$56^{\text {ème }}$ Congrès de la SFMBCB, 02009 (2011)

DOI: $10.1051 / \mathrm{sfmbcb} / 20115602009$

(C) Owned by the authors, published by EDP Sciences, 2011

\title{
Apport de la reconstitution tridimensionnelle (logiciel MVS) dans l'avulsion des troisièmes molaires mandibulaires en rapport avec le canal dentaire inférieur
}

\author{
Fabris M, Belangeon T, Paris M, Bodard AG, Veyre S, Coudert JL
}

\author{
Service de Consultations et de Traitements dentaires, Département de Chirurgie buccale, \\ Hospices civils, Lyon, France \\ matthieufabris@orange.fr
}

Pour la bonne réalisation de l'avulsion de la troisième molaire mandibulaire il faut un bon diagnostic. Celui-ci comporte une anamnèse précise du patient, un examen clinique bien mené associé à des examens complémentaires d'imagerie (Teman et al 2004). Les difficultés rencontrées viennent essentiellement de sa relation anatomique intime avec le paquet vasculonerveux alvéolaire inférieur (Engström et Sagne 1980) pouvant entraîner non seulement des hémorragies per ou post-opératoires mais également des lésions nerveuses transitoires ou permanentes du type paresthésie ou anesthésie labio-mentonnière. Selon la littérature, l'incidence de ces dommages nerveux est comprise entre 0,5 et $8 \%$. Si la radiographie panoramique est un examen indispensable au chirurgien avant l'avulsion d'une dent de sagesse, celui-ci ne renseigne que partiellement sur la position exacte des racines par rapport au canal dentaire inférieur dans le sens vestibulo-lingual. Dans ces cas, l'utilisation des coupes CT-scan constitue une aide précieuse pour la localisation des apex dentaires car elles fournissent des informations dans les trois plans de l'espace (Lacan 1995).

L'étude porte sur 500 cas de dents de sagesse mandibulaires, observées entre octobre 2008 et décembre 2009 dans le Département de Chirurgie buccale du SCTD des Hospices civils de Lyon, pour lesquels les racines présentaient des rapports étroits avec le canal dentaire inférieur, détectés à l'examen orthopantomographique. Pour l'ensemble de ces cas, un examen tomodensitométrique a été prescrit pour déterminer la position exacte du canal mandibulaire par rapport aux racines de la dent. Les fichiers des coupes d'acquisition parviennent sous forme d'un CD-Rom au format DICOM. Les reconstructions tridimensionnelles sont réalisées grâce au logiciel MVS. Cette reconstitution $\mathrm{du}$ site d'avulsion est surfacique. Cette reconstitution $3 \mathrm{D}$ permet d'adapter la technique chirurgicale, notamment pour la voie d'abord, et précise la position vestibulaire ou linguale du canal mandibulaire par rapport aux racines dentaires. Cela permet d'anticiper le mouvement de l'élévateur afin de luxer la racine pour éviter le contact des racines avec le canal. Ainsi, l'opérateur peut transférer, au moment de l'intervention, le virtuel au réel.

De toutes les interventions réalisées, aucune n'ont conduit à une paresthésie ou anesthésie temporaire ou définitive labio-mentonnière. La reconstruction tridimensionnelle permet d'adapter le geste chirurgical et de supprimer la lésion du paquet vasculo-nerveux et les lésions nerveuses subséquentes 